\title{
Iris Recognition System (IRS) Using Deep Learning Technique
}

\author{
Sue Chin Yow and Ahmad Nazri Ali* \\ School of Electrical and Electronic Engineering, Universiti Sains Malaysia, \\ Engineering Campus, 14300 Nibong Tebal, Pulau Pinang, Malaysia \\ ${ }^{*}$ Corresponding author: nazriali@usm.my
}

Published online: 31 December 2019

To cite this article: Sue Chin Yow and Ahmad Nazri Ali. (2019). Iris recognition system (IRS) using deep learning technique. Journal of Engineering Science, 15(2), 125-144, https://doi.org/10.21315/jes2019.15.2.9

To link to this article: https://doi.org/10.21315/jes2019.15.2.9

\begin{abstract}
Identity recognition through human iris organ is claimed as one of the famous biometric techniques due to its reliability promising higher accurate return as compared to other traits. Reviewing past literatures, poor imaging condition, low flexibility of model, and small size iris images dataset are the limitations needing solutions. In this paper, a proposed algorithm development flow and systematic analysis has been conducted to achieve high efficiency in the iris recognition task. A transfer learning method that does not involve iris segmentation phase is proposed to capitalise pre-trained Convolutional Neural Network (ConvNet) model introduced in the ImageNet Large Scale Visual Recognition Competition (ILSVRC) on iris recognition system. Both data augmentation and Bayesian optimisation are also involved in optimising the network and prevent it from overfitting. Simulation results showed the transferability of a pre-trained model on new target task is improved and meanwhile, the high recognition rate of the algorithm on small-size Institute of Automation, Chinese Academy of Sciences (CASIA) Iris-Interval V1 iris image dataset is achieved.
\end{abstract}

Keywords: deep learning, ConvNet, transfer learning, iris recognition

\section{INTRODUCTION}

Iris recognition is a type of biometric technology that enables a person to be authenticated automatically via his criteria through machines. Compared to other biometric traits, iris possess lesser false acceptance and rejection rate due to its high in universality, uniqueness, permanence, permanency, and stability. ${ }^{1}$ Informative features of iris are being extracted from the raw image and those features are classified based on criteria. Correct prediction on the label indicates successful iris recognition. In the literature, ideal image acquisition conditions are 
assumed in most recognition systems in order to get a high recognition rate. ${ }^{2}$ Most of the open source iris database in previous time are captured using near infrared (NIR) camera in close distance, along with the look and stare constraints condition so that the pigmentation of the iris image is revealed and clear texture to obtain. It saves a bundle of engineers' time in filtering the noise.

In Libor Masek analysis, $83 \%$ of the Institute of Automation, Chinese Academy of Sciences (CASIA) database images were segmented correctly while the Lion's Eye Institute (LEI) database has only $62 \%$ of the images segmented successfully due to poor imaging conditions. ${ }^{3}$ LEI database that imaging under natural light is containing specular reflections and difficult to segment iris region as compared to CASIA database that uses NIR light for illumination in image acquisition.

Besides that, flexibility in terms of accepting the appropriate input image is seeking for improvement. The ready solution is through the effort made on both hardware and software, purposely to eliminate the unwanted features and highlighting the important details of the image captured. Most of the time, the system will not get any input if the most desired condition is not met. ${ }^{4,5} \mathrm{In}$ this case, most of the hand-crafted algorithm unable to be simply adapted to new conditions without fine tuning on software parameters.

However, the growing interest of deep learning (DL) and computer vision in image processing indicate that feature extraction using Convolutional Neural Network (ConvNet) effectively extracting discriminative visual features iris images and eliminating the laborious feature-engineering task. The limitation of traditional iris recognition system to process iris images that captured in unconstraint environments is breakthrough. Despite the robustness of DL in training machine to solve various tasks, the most important limitation lies in training dataset size and computational power in generating a powerful algorithm.

The main goal of this paper is to overcome the poor robustness of classical hand-crafted feature extraction method in iris recognition system (IRS). Transfer learning are fully utilised to increase flexibility of model on new environment. Apart from this, technique like data augmentation and Bayesian optimisation also been suggested for limited dataset size and computational power purposes. ${ }^{6,7}$

The strategies of this paper are as follows:

1. First, this project manages to eliminate the iris segmentation phase since in conventional IRS, its' failure can indirectly affect the recognition rate. 
2. Second, transferability of pre-trained ConvNet model can be tested using support vector machine (SVM) classifier with transfer learning technique.

3. Third, a high-performance ConvNet model that able to run IRS task is developed using transfer learning technique.

4. Forth, image augmentation, Bayesian optimisation and layer freezing techniques are being applied for better model performances.

Almost all classical machine learning (ML) in IRS involves these elementary steps, including image acquisition, pre-processing, features encoding, and matching for user authentication or identification. ${ }^{5}$ Iris segmentation in Figure 1 is the most crucial part in the IRS since the feature of the iris has to be extracted correctly to enable achieve high accuracy in the matching stage.

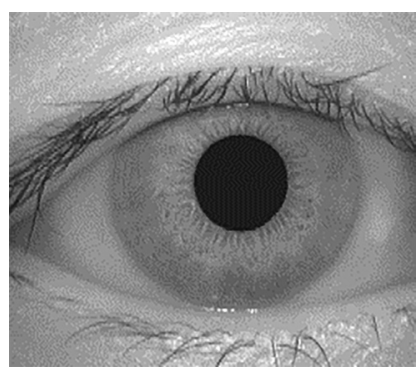

(a)

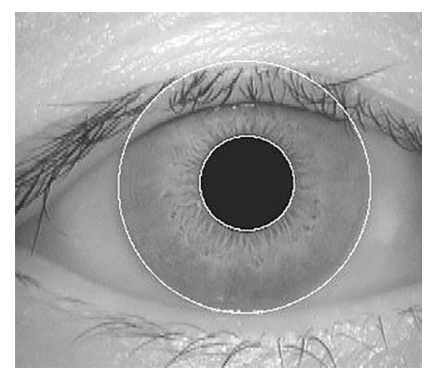

(b)

Figure 1: (a) Original iris image and (b) segmented iris image. The boundary of iris is segmented after applying Circular Hough Transform method. ${ }^{3}$

DL provides a flexible and scalable ML method that requires no more constraint on the feature extraction while training the algorithm, especially in iris recognition algorithm development. However, certain research results show that DL can underperform as compared to classical ML techniques sometimes. This result is difficult to predict and explained mathematically as DL is a black box concept. Thus, this ignites the curiosity and spirit of researchers in investigating the root cause of failure, and discover a new neural network (NN) algorithm, tend to get high accuracy and precise rate for the specific systems. As DL becomes the technology trend, many research journals had been proposed and published, aimed to study DL from a different perspective. Instead of using a conventional algorithm for iris segmentation, researchers proposed a new algorithm and test on their effectiveness on both NIR and visible light database. Semi-parallel Deep Neural Network (SPDNN) and IrisDenseNet are developed and both of them perform well in the iris segmentation region, even with a low-resolution image. ${ }^{8,9}$ 
The standard reference for ConvNet is from 1999 by LeCun et al., "Object Recognition with Gradient-Based Learning". ${ }^{10}$ However, the first ConvNets idea that revealed, called neocognitron. It is proposed by Kunihiko Fukushima in the 1980 s, which is a hierarchical multi-layered artificial neural capable of the robust visual pattern. ${ }^{5}$ When coming to the world of DL, ConvNets are one of the most popular and high usage categories of NN, especially for high dimensional data, a field or matrix (e.g. videos ori). ${ }^{11}$ ConvNet designed specifically for image recognition and classification problems. ${ }^{12}$ ConvNet is a sequence of layers of neural nets, wherein each hidden layer transforms one volume of activations to another through a differentiable function. The three type layers that build up ConvNet are the convolution layer, pooling layer, and fully connected layer. As compared to the previous regular NN layer, the convolution layer does not employ connection weights and a weighted sum. Figure 2 shows the existing ConvNet published in ImageNet Large Scale Visual Recognition Competition (ILSVRC), an annual competition in visual recognition. ${ }^{13}$

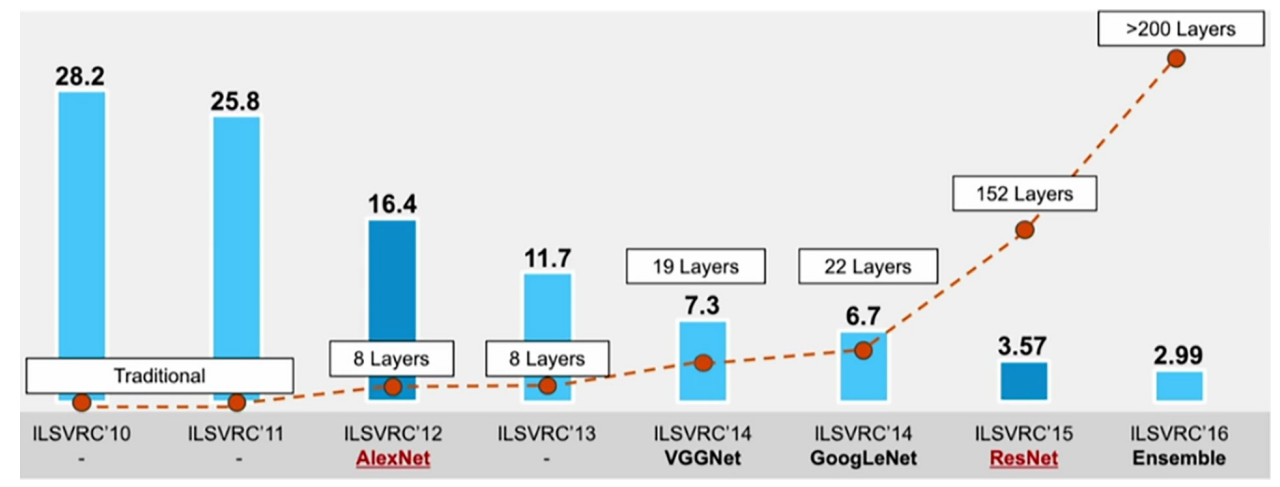

Figure 2: Recent ConvNets proposed in ILSVRC.

The central concept of transfer learning is to employ the existing successful pre-trained NN model to "transfer" their learning to a more simplified problem. During this transfer training, some hidden layers can be frozen in order to decrease computation time. There are multiple ways in utilising transfer learning techniques. It can make use of the layer in pre-trained ConvNet model to extract the features of image and train the SVM classifier with that features for further image classification. ${ }^{14}$ The flow is illustrated in Figure 3.

The other way is to take advantage of the ready architectures of the pretrained ConvNet model. Simply chopped last few layers since the shallower layers of a ConvNet are essentially learning how the features of the image look like and only the last one or two layers of a ConvNet are performing the most complex 
tasks of summarising and categorising the vectored image data into their specific class that they are supposed in. Re-train the model with target database instead of scratch is demonstrated in Figure 4. ${ }^{15}$

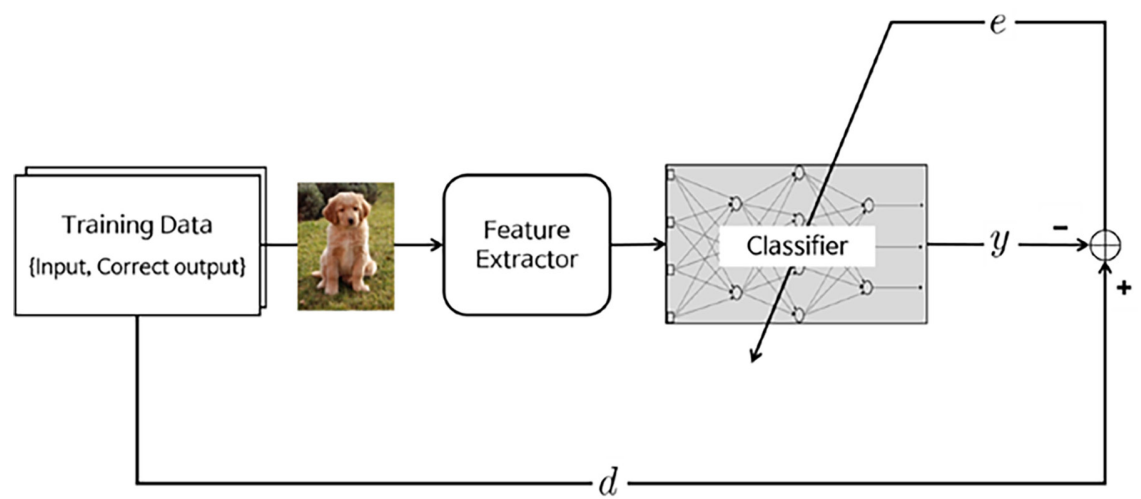

Figure 3: Image feature extraction by ConvNet model and trained SVM classifier for image classification. ${ }^{15}$

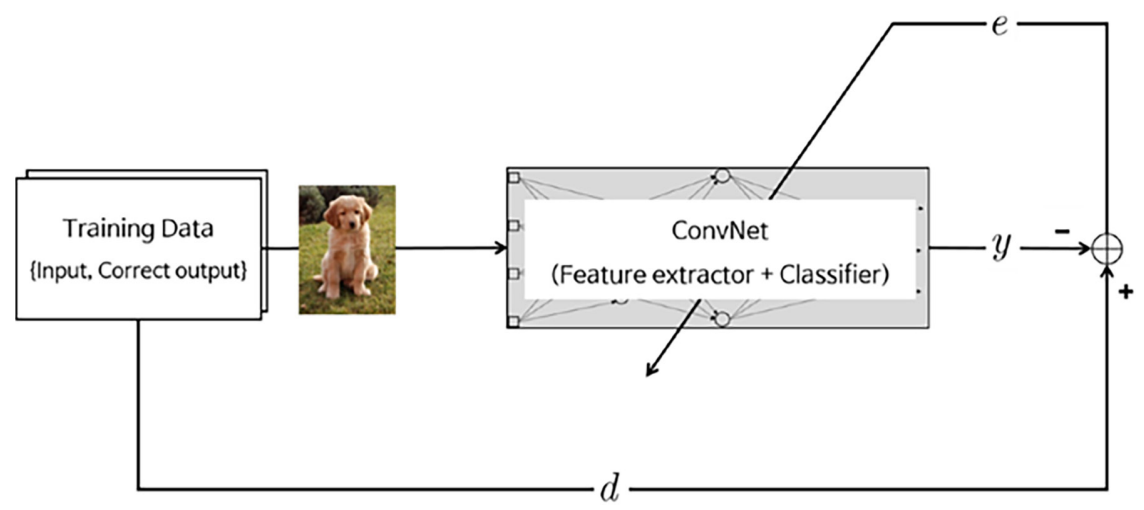

Figure 4: Re-train entire ConvNet model for image classification. ${ }^{15}$

\section{MATERIALS AND METHODS}

This is a fully software development project on training IRS algorithms. The algorithm is developed by training with the target dataset using DL technique and the proposed development workflow is shown in Figure 5. The results are tabulated and performances are evaluated. 


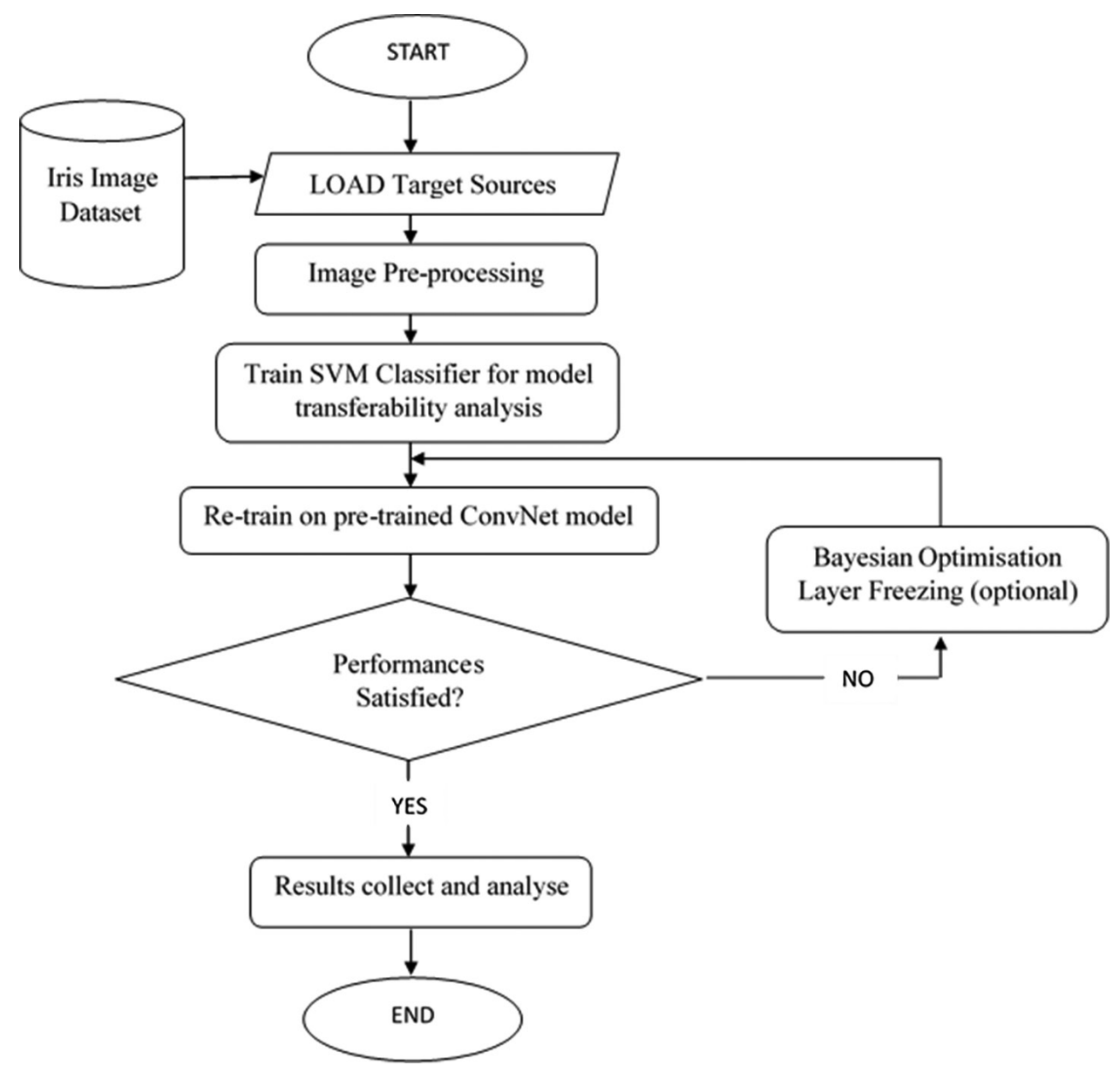

Figure 5: Project workflow.

The assessment is done on Intel Core i5 $2.2 \mathrm{GHz}$ machine with $8 \mathrm{~GB}$ installed RAM employing Window 8.1. Meanwhile, MATLAB R2019a riches with ML libraries and experts in processing matrix calculation was used to develop the image processing algorithm in this project. Deep Learning Toolbox with a pretrained model, AlexNet and DenseNet201 support packages were installed.

CASIA-Iris-Interval $\mathrm{V} 1$, an iris database that contains the complete eye part, was used as a target dataset along with this iris recognition project for algorithm simulation and development. It is one of the oldest publicly available systems for evaluation of the iris biometric modality. This version contains 756 iris images from 108 human subjects. The images in CASIA-Iris-Interval V1 have 
been captured using a homemade iris camera, with eight circularly fitted NIR 850 $\mathrm{mn}$ illuminators. ${ }^{2}$ All the images are stored in the format of BMP at $280 \times 320$ resolution.

Training a network and making predictions on new data require images that match the input size of the network. Resize all the images in the CASIAIris-Interval-V1 to fixed dimensions that capable with the architecture of the model selected. For AlexNet and DenseNet201, the input images were resized to $227 \times 227 \times 3$ and $224 \times 224 \times 3$, respectively. Instead of image cropping to adjust image size, resizing method can prevent loss of informative features for further processing.

In all algorithm training process, all the datasets are equally distributed into three main sets with a ratio of 0.42:0.29:0.29, which are further labelled as training, validation, and testing accordingly. For CASIA-Iris-Interval V1, 108 users have a total of seven original iris image each and after allocation, it was divided into three sessions, contain training session (three images), validation session (two images), and testing section (two images). Hence, a total of 756 images was sorted to 324 training images data, followed by 216 validation images data and 216 testing images data. Both training datasets and validation datasets are used during NN training. The training dataset adjusts the weight of NN while the validation dataset prevents the NN from overfitting. Additionally, a testing dataset is only used for confirming the actual predictive power of the well-trained network.

Data augmentation is one of the solutions for small size dataset in DL training by increasing the variety of training samples. ${ }^{6}$ Its strategies can be in terms of flipping, scaling, cropping, shearing, rotation, and translation. These operations on images can be performed separately or combined together. ${ }^{11}$ For instance, rotation after cropping. In each iteration, training data in a mini batch is transformed based on a random combination of transformation strategies and the transformed images are not stored in memory. Hence, the actual number of training samples does not change via augmentation. The training data were perturbed in every epoch and the network trained using a slightly different training dataset copies in every epoch. Parameters for each strategy are tabulated in Table 1.

Instead of training from scratch, transfer learning is a popular method that fine-tunes a pertained network with the target dataset and allows to build an accurate model in a timesaving way. ${ }^{16}$ In this paper, transfer learning is utilised for two purposes. One of them is testing for the transferability of the pre-trained model and another one is using the pre-trained model as framework, transfer its' ability and learning for new target task. 
Table 1: Parameters for data augmentation.

\begin{tabular}{llc}
\hline Strategy & Definition & Range/parameter \\
\hline Scale & Range of uniform (isotropic) scaling & $0.5-2.0$ \\
Rotation & Range of rotation, in degree & \pm 10 \\
X-axis translation & Range of horizontal translation, in pixels & \pm 30 \\
Y-axis translation & Range of vertical translation, in pixels & \pm 30 \\
\hline
\end{tabular}

Since the selection of the suitable pre-trained model is a major part in applying transfer learning technique, the transferability of the pre-trained model on CASIA-Iris-Interval V1 dataset can be pre-tested using trained SVM classifier. The extracted features of the training dataset were extracted using activation on desire feature layer on either AlexNet or DenseNet201 pre-trained model. The extracted features of training datasets with 324 samples were then used to train a multiclass SVM classifier. The test features were then extracted from the test sample sets, passing to the classifier and measure the accuracy of the trained classifier for its accuracy rate. The classification accuracy results of SVM classifier represent the transferability of model.

The steps of using transfer learning in re-training the entire ConvNet model are listed as follows:

1. Step 1: Choose on pre-trained ConvNet model and iris database.

2. Step 2: Optimise hyperparameters using Bayesian optimisation.

3. Step 3: Select the number of layers freeze (optional).

4. Step 4: Retrain the ConvNet model with optimising hyperparameters.

5. Step 5: Collect results in the form of data and figures.

Since the target dataset is task different from the base training dataset, it needs to find a balance on the number of layers to train and freeze respectively. The model can be overfitting if trained too deep or the model unable to learn the features of the target dataset if it remains at the shallow end. In this case, this project was trained by freezing different neural layers without updating the weight on frozen layers and the last fully connected layer was replaced with the layer matching the number of classes in the target dataset, which is 108 for CASIA-IrisInterval V1. The proposed algorithm also eliminates the step of iris segmentation and avoids error in the iris segmentation phase that may reduce the overall iris recognition rate. Along the algorithm development process, the network training was stopped when one of the following criteria was met: 
1. the validation accuracy remains unchanged, or

2. the training accuracy reaches a plateau and no longer improving, or

3. overfitting occurs.

By taking posterior evaluations into account, it updates the surrogate function along with the feedback of the objective function when choosing for the hyperparameter to evaluate next. The objective function indicates how well a set of hyperparameters performs on the validation set. ${ }^{7}$ There are some journals published about the implementation of Bayesian optimisation to improve the performances of Deep Convolution Network in object detection ${ }^{17}$ and text prediction but have no journal that related to the application of Bayesian optimisation in IRS up to now. ${ }^{18}$

Table 2 shows the range of a set of variables that looking for optimisation. The three hyperparameters of the model that need to be optimised are learning rate, momentum, and $\mathrm{L} 2$ regularisation. The objective function for the Bayesian optimiser is created, along with training and validation data as inputs. Then, the classification error on the validation set was returned after the objective function was trained on ConvNet. Based on the validation error rate, the best model that chosen at the final has to be tested for generalisation error.

Table 2: Range of hyperparameters variables.

\begin{tabular}{llc}
\hline Variable & Definition & \multicolumn{1}{c}{ Range } \\
\hline Initial learning rate & $\begin{array}{l}\text { Control how quickly the weights are } \\
\text { adjusted with respect the loss gradient. }\end{array}$ & {$\left[1 \times 10^{-2}-1\right]$} \\
$\begin{array}{l}\text { Stochastic gradient descent } \\
\text { (SGD) momentum }\end{array}$ & $\begin{array}{l}\text { Accelerate SGD in the relevant direction } \\
\text { and dampens oscillation. }\end{array}$ & {$[0.80-0.98]$} \\
L2 regularisation strength & $\begin{array}{l}\text { Act of modifying a learning algorithm } \\
\text { to avoid overfitting. }\end{array}$ & {$\left[1 \times 10^{-10}-1 \times 10^{-2}\right]$} \\
\hline
\end{tabular}

Both classification accuracy and error are reported to know how prone a model is to make mistakes. Quantitatively, the performance of an algorithm was observed in kind of data. In MATLAB, the accuracy rate is calculated based on the mean number of correct predictions. Mathematically, the equations that involved in calculating classification accuracy rate and classification error are shown in Equations 1 and 2, respectively.

$$
\begin{aligned}
& \begin{array}{l}
\text { Classification } \\
\text { accuracy rate }(\%)
\end{array}=\left(\sum_{i=1}^{N} \frac{\text { Number of correct predictions }}{\text { Total number of predictions }} \times 100\right) / N \\
& \text { Classification error }=\frac{\text { Number of correct predictions }}{\text { Total number of predictions }}
\end{aligned}
$$


Confidence levels and confidence intervals are a way of quantifying the uncertainty of estimating the performance of a machine learning algorithm on unknown data. By using the central limit theorem, it can measure how much confidence can place in measurements from the samples. Wilson score interval method is applied and the formula used for calculating the confidence interval is shown in Equation 3 as follows:

$$
p=\hat{p} \pm \sqrt[z]{\frac{\hat{p}(1-\hat{p})}{n}}
$$

where $\hat{p}$ is classification error, $z$ is sigma value corresponding to level of confidence, and $n$ is total number of prediction samples. The $95 \%$ confidence level was selected to examine performances of the classification accuracy in this paper and hence, value is \pm 1.96 .

\section{RESULTS}

This section is organised by first displaying the results for the image after pre-processing. It then discussed on the transferability of the pre-trained ConvNet model by doing an evaluation on classification accuracy. Afterward, evaluation on the performance of algorithms regardless of data augmentation are done. To optimise hyperparameters, Bayesian optimisation is applied and training results were displayed in tabular form. Follow by this, there is discussion about the impact of layer freezing techniques in solving the limitation on computational power. Finally, evaluation and analysing the comparative performances of each algorithm in both quantitative and qualitative insight are discussed.

In MATLAB, training image datasets were automatically augmented with the constraint hyperparameter range in every training batch, it does not store in memory. Mini batch of the augmented training image is reviewed and evaluated by naked eyes. Figure 6 shows mini batch of training images taken from CASIA-IrisInterval V1 Dataset and undergo non-augmentation and augmentation. For data augmentation, the images are obviously being transformed and viewed in different perspectives. From the figure displayed, it can be verified that the training images from CASIA-Iris-Interval-V1 dataset are well augmented under constrained hyperparameters set in terms of scaling, rotation, and pixel translation. 


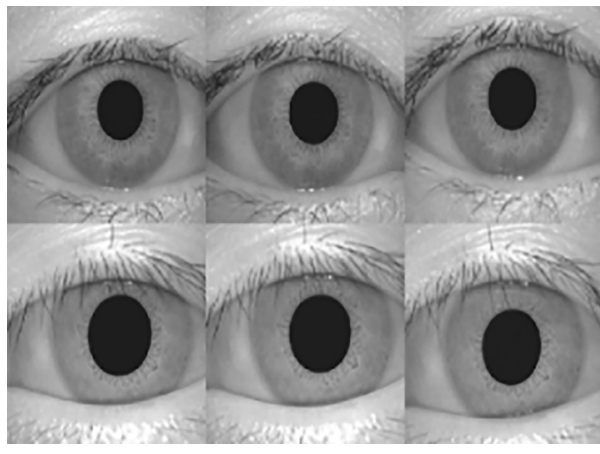

(a)

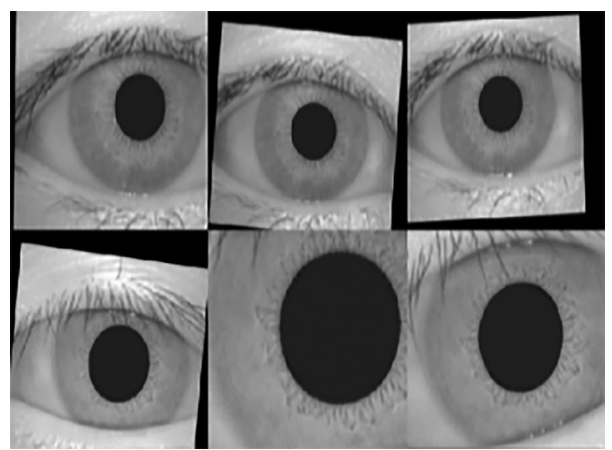

(b)

Figure 6: Mini batch of iris images (a) without data augmentation and (b) with data augmentation. ${ }^{19}$

ConvNets model generates an algorithm that enables a machine to differentiate important features of the target dataset and train on them without any human supervision. Figure 7 displays the feature maps extracted from the first convolutional layers of the AlexNet network. The channels in earlier layers learn simple features like edge and pattern of the iris.

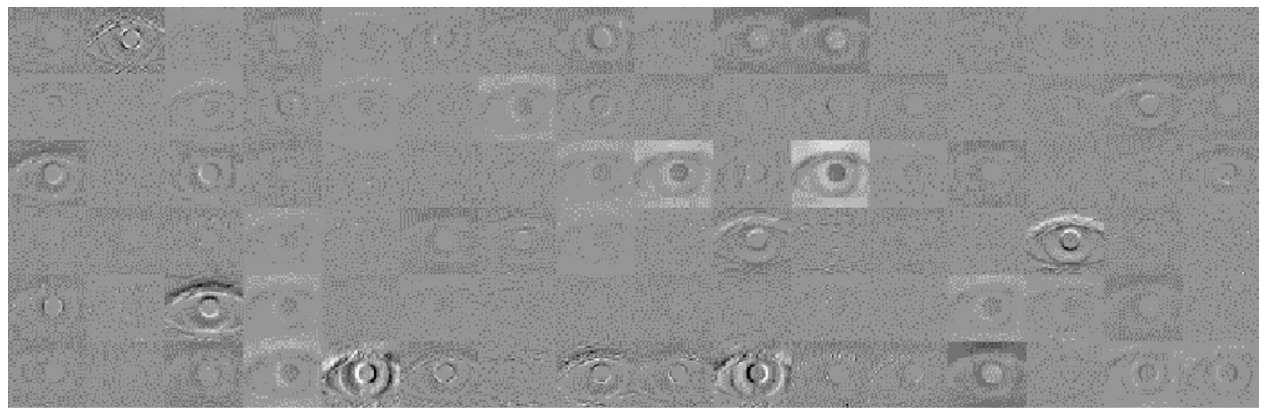

Figure 7: Feature layer of the first convolutional layer in AlexNet model.

However, the selection of suitable pretrained source model can greatly reinforce the transferability of transfer learning. AlexNet and DenseNet201 pretrained models were selected in this paper. Table 3 tabulates the accuracy rate of the SVM classifier after training using the activation feature layer from different layers. AlexNet pretrained model exhibits good result with $87.43 \%$ classification accuracy rate on the third convolution layer. Apart from that, classification accuracy rate had achieved the highest rate at $97.07 \%$ over 756 samples from CASIA-Iris-Interval-V1 when classifier trained on the 600th layer of DenseNet201 pretrained model. Since both of the pretrained networks demonstrated great results 
on the classification accuracy, it indicates that both pretrained AlexNet model and DenseNet201 model able to adapt well on IRS and have potential to train with target database for better accuracy rate. In order to achieve a higher accuracy rate, the pretrained model can be retrained on target dataset sources and update the weight of NN.

For evaluating algorithm performance, only AlexNet model is selected to test on the algorithm performances for the impact of data augmentation. From the view of qualitative, the accuracy rate is higher for the model that is trained with an augmented training dataset. According to the numerical result in Table 4, there are $5.92 \%$ higher in overall accuracy rate on the model that was applying the augmented training dataset method.

Table 3: Transferability of pretrained ConvNet model on CASIA-Iris-Interval V1 dataset.

\begin{tabular}{llcc}
\hline Model & Layer's name & Layer's position & Classification accuracy (\%) \\
\hline Pretrained & conv3 & 10th & 87.43 \\
AlexNet & conv4 & 12th & 86.90 \\
& conv5 & 14th & 82.14 \\
& Pretrained & 200th & 89.95 \\
DenseNet201 & conv4_block38_0_bn & 400th & 92.99 \\
& conv5_block18_0_bn & 600th & 97.07 \\
\hline
\end{tabular}

Table 4: Performance analysis on AlexNet model regardless of data augmentation.

\begin{tabular}{llcc}
\hline Model performance & & $\begin{array}{c}\text { Non-augmented training } \\
\text { dataset }\end{array}$ & $\begin{array}{c}\text { Augmented training } \\
\text { dataset }\end{array}$ \\
\hline Accuracy (\%) & Training & 100.00 & 98.15 \\
& Validation & 86.57 & 96.30 \\
& Test & 84.72 & 94.44 \\
& Overall & 91.80 & 97.72 \\
Loss & Training & 0 & 0.0185 \\
& Validation & 0.1343 & 0.0370 \\
& Test & 0.1528 & 0.0556 \\
& Overall & 0.0820 & 0.0228 \\
\hline
\end{tabular}


The training plot in Figure 8 reveals that overfitting was happening on the model with the non-augmented training dataset. The overfitting occurred when the validation accuracy is higher than training accuracy after an epoch of 10 . Meanwhile, there are up to $13.43 \%$ high difference between the training accuracy rate and validation accuracy rate which also indicates that the model is overfitting intensively. However, no overfitting occurred in Figure 9, which trained with the augmented training dataset. The training accuracy rate of the model increases gradually as the epoch increases.

The reason is there were too few examples given to training when the model was training using a small size dataset, and finally resulting in a poor generalisation performance model. Meanwhile, augmentation on training data had made the training data infinite by enriching the training data and generating more dissimilar training sample examples via random transformation of existing one. This can be concluded that the accuracy obtained on a new dataset depends on both the quality and quantity of the training dataset.

Bayesian optimisation was used to find out the optimum values for the hyperparameters of the model. The three hyperparameters of the model that need to be optimised are learning rate, momentum, and L2 regularisation. This part discusses about the results of Bayesian optimisation on the AlexNet model and the DenseNet201 model that trained on the target dataset. After applying Bayesian optimisation on AlexNet model, the 8th iteration presents the least validation error of 0.20833 with 0.001 of initial learning rate value, 0.90305 of momentum value, and $1.61 \times 10^{-6} \mathrm{~L} 2$ regularisation value. Besides, for DenseNet201 model, the 4th iteration presents the least validation error of 0.037037 with 0.057675 of initial learning rate value, 0.8295 of momentum value, and $9.11 \times 10^{-8} \mathrm{~L} 2$ regularisation value. From the view of data, the DenseNet201 model performed lesser iteration than the AlexNet model in selecting hyperparameters as the maximum objective function evaluation time was achieved. Instead of less evaluation, the DenseNet201 model still has a lower estimated objective function value which is 0.037331 as compared to 0.22505 in the AlexNet model. Although the DenseNet model with a deeper layer has a higher classification accuracy rate, the computational time estimated to train on the target dataset is massive, which is $8,329.8878 \mathrm{sec}$. This is approximately six times higher than the AlexNet model, which needs $1,195.15 \mathrm{sec}$. Hence, different sets of hyperparameters show an obvious different impact on the performance of the model trained. Search for possible hyperparameters by applying Bayesian optimisation made training progress effective and efficient instead of using trial and error method. 


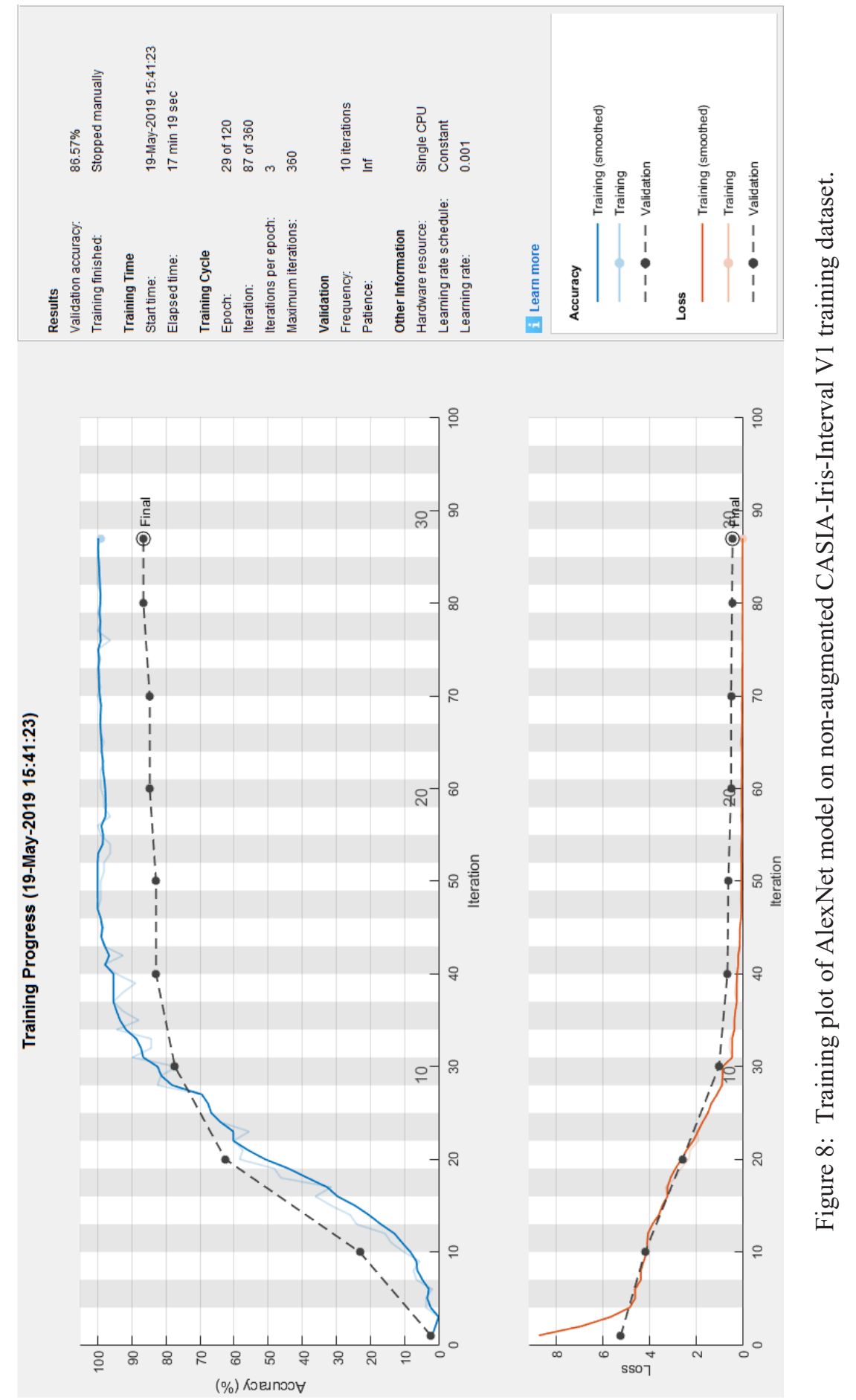




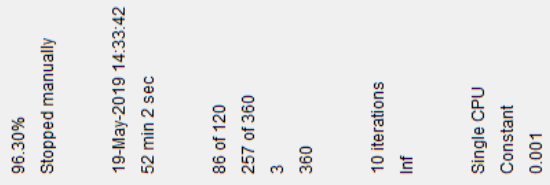

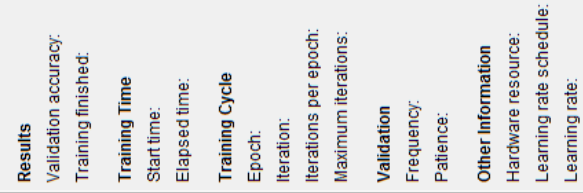
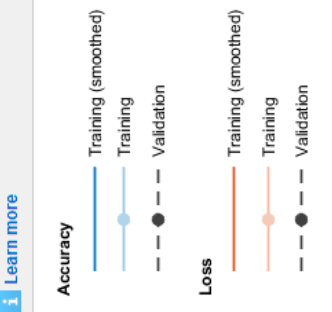

苞
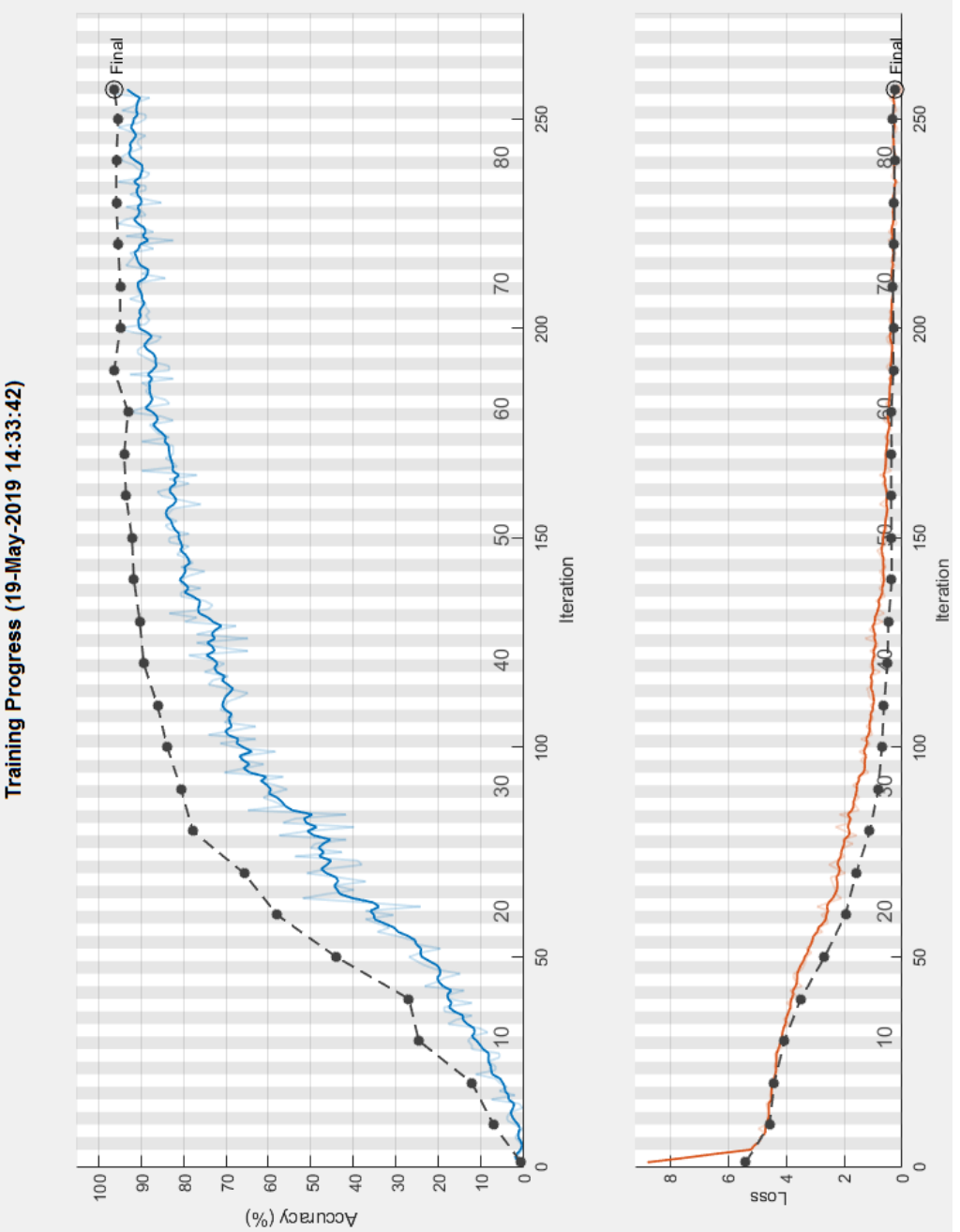

> 
Along the project, there are few challenges encountered and one of them is the system hanging when there is a re-training process on the entire DenseNet201 model. In this case, layer freezing technique is utilised to reduce the burden of the system on computational works. Table 5 shows the impact of the layer freezing on both the computational time and the accuracy rate. As the layer freezing decreases, the computational time and accuracy rate of the DenseNet201 model increases. It indicates that when more layers are left for re-training, more computational power needed for weight updating and more accuracy the result getting. There are significant raising in computational time, which is from approximately $1 \mathrm{~h}$ training period to $15 \mathrm{~h}$ training period when the layer froze decrease from 600th layer to 200th layer.

Table 5: Recognition accuracy rate versus layer freezing on DenseNet201 model with CASIA-Iris-Interval V1 dataset.

\begin{tabular}{llll}
\hline $\begin{array}{l}\text { Layer freezing } \\
\text { (out of 709 layer) }\end{array}$ & $\begin{array}{l}\text { Computational time } \\
\text { (hh:mm:ss) }\end{array}$ & Performance & Accuracy rate (\%) \\
\hline 600 th & $01: 15: 03$ & Validation & 94.44 \\
& & Test & 92.13 \\
& \multirow{2}{*}{$02: 01: 02$} & Overall & 96.16 \\
400 th & & Validation & 96.30 \\
& & Test & 96.30 \\
& \multirow{2}{*}{ 15:43:48 } & Overall & 97.88 \\
& & Validation & 97.69 \\
& & Test & 98.15 \\
\hline
\end{tabular}

Along with the algorithm training progress, both AlexNet and DenseNet201 algorithms that possess the highest accuracy rate in classifier CASIA-Iris-Interval V1 are being selected. Table 6 tabulates the specification details and performances of them independently. As compared to the AlexNet, the DenseNet201 model is a complex NN that possess 201 weight layer depth and thus, the computational time needed for training progress is much higher in the DenseNet201. Next, $98.81 \%$ of the accuracy rate in the DenseNet201 model is slightly more than the accuracy rate of $97.22 \%$ in AlexNet. There is a trade-off between complexity and accuracy rate. However, AlexNet has much more difference between overall accuracy and test accuracy than DenseNet201. This represents that AlexNet is much weaker in doing prediction on a new test set that unseen before. To evaluate the performance of accuracy from a statistical perspective, AlexNet has a 95\% probability that the test error rate will fall within a range of 0.0250 to 0.0861 . Meanwhile, DenseNet201 has an interval range of 0.0005 to 0.0365 . 
Interestingly, both developed algorithms using different ConvNets model have a low precision rate on the registered user class labelled "104" in CASIA-IrisInterval V1 datasets. Retrained DenseNet201 model has four out of seven images (51.14\%) predicted correctly in class " 104 " while for retrained AlexNet model, it has only three out of seven images (42.86\%). It can be explained that overall accuracy is mostly affected by low precision in the user class of "104". After checking on the iris images respective user, it found that there is a bit difference between the training dataset, validation dataset and test dataset. This can be due to training dataset has an iris image with smaller pupil's diameter as compared to validation datasets and testing datasets. The weight parameter tuned based on the features extracted from the training dataset during the training stage affects the probability in prediction the class of the respective iris images. To solve this problem, shuffle images of dataset before training is suggested.

Table 6: Specification details and performances of retrained model based on proposed technique.

\begin{tabular}{lcc}
\hline Retrained model specifications & AlexNet & DenseNet201 \\
\hline Complexity (layer of depth) & 8 & 201 \\
Image augmentation & Yes & Yes \\
Bayesian optimisation & Yes & Yes \\
Layer freezing (opt.) & None & 200th out of 709 \\
Computational time (hh:mm:ss) & $00: 51: 54$ & $15: 43: 48$ \\
Processing time (s) & 0.125955 & 1.467658 \\
Overall accuracy (\%) & 97.22 & 98.81 \\
Test accuracy (\%) & 94.44 & 98.15 \\
95\% confidence interval of test error & {$[0.02500 .0861]$} & {$[0.00050 .0365]$} \\
Lowest precision rate & $42.86 \%$ on user no. “104” & $57.14 \%$ on user no. “104” \\
\hline
\end{tabular}

\section{CONCLUSION}

In this project, the IRS with the proposed technique workflow was successfully developed using MATLAB code. The developed system able to recognise the 108 class of input image inserted from target dataset, CASIA-IrisInterval V1 dataset. Proposed training method with transfer learning able to take existing algorithms as a benchmark instead of scratch. Before the algorithm retraining process, the model is pre-test using an extracted feature on the SVM classifier and does classification on the target dataset. This method aid to identify the transferability and select the suitable model. Based on the results, both AlexNet 
and DenseNet201 models able to achieve more than $80 \%$ accuracy and hence, it indicates the models' high transferability on the IRS. Data augmentation technique helps to transform original images of the target dataset and provides more different samples for the training procedure to solve the limited data size problem and help prevent the algorithm from over-fitting. By data augmentation works on training datasets, the overall accuracy rate of iris images on trained AlexNet model can be increased from $91.80 \%$ to $97.72 \%$. In this case, both poor imaging conditions of input and flexibility of model can be solved.

Moreover, optimum values for hyperparameters were successfully found through the Bayesian optimisation technique. Coupled with those proposed techniques, transferability of the pretrained model is improved in this project. To overcome the hardware limitation, layer freezing techniques is applied to reduce the burden of the computational power of the model especially for large size model like DenseNet201 with 201 depth layers. When there are more layers had been frozen, less computational time is needed and less accurate the recognition rate.

Both AlexNet and DenseNet201 models able to adapt in IRS with an accuracy rate of $97.22 \%$ and $98.81 \%$, respectively. However, there is trade-off between the complexity of model with the computational time and algorithm processing time. To be justified, DenseNet201 is a computationally intensive network with its rich dense connections, allowing neurons to interact easily and achieving better recognition accuracy than AlexNet in the iris recognition task. Architecture of AlexNet model is small and may not able to fully capture the discriminative visual features in iris images in Nguyen and colleagues. ${ }^{14}$

In conclusion, transfer learning able transfer knowledge for features extraction and classification on a new task. The proposed methodology able to solve poor imaging condition, flexibility of models, and limited dataset size declared in section before. All the procedures are crucial in developing a well fit $\mathrm{NN}$ that able to solve multiple tasks.

\section{SUGGESTION FOR FUTURE IMPLEMENTATION}

Other than the selected criteria, there are still lots of other outstanding published networks such as Inception, GoogleNet, and NaSNet-Large present. This project can be extended to adapt and test on other available models on the different public iris datasets. To minimise the trade-off matter, the model reduction technique can be considered to eliminate redundant layers and neurons, thus, decrease the size of the network. Hardware with high computational power needed 
to be taken into account when training on a huge ConvNet network. Besides, the size of the final trained model has to concern when it is designed to deploy a model to run locally on mobile. A portable IRS can be realised by integrating a powerful and memory-efficient network using a transfer learning technique onto a handy device.

\section{ACKNOWLEDGEMENTS}

A portion of the images in this paper is from the Institute of Automation, Chinese Academy of Sciences (CASIA) database, which is freely available online. ${ }^{19}$

\section{REFERENCES}

1. Sabhanayagam, T., Venkatesan, V. P. \& Senthamaraikannan, K. (2018). A comprehensive survey on various biometric systems. Int. J. App. Eng. Res., 13, 2276-2297.

2. Al Rifaee, M. (2014). Unconstrained iris recognition. PhD diss., De Montfort University.

3. Masek, L. (2003). Recognition of human iris patterns for biometric identification. BEng diss., University of Western Australia.

4. Cruz, F. R. G., Hortinela, C. C., Redosendo, B. E., Asuncion, B. K. P., Leoncio, C. J. S., Linsangan, N. B. \& Chung, W. -Y. (2016). Iris recognition using Daugman algorithm on Raspberry Pi. 2016 IEEE region 10 conference (TENCON). New Jersey, USA: Institute of Electrical and Electronics (IEEE), 2126-2129, https://doi.org/10.1109/ TENCON.2016.7848401.

5. Kunik, Z., Bykowski, A., Marciniak, T. \& Dąbrowski, A. (2017). Raspberry Pi based complete embedded system for iris recognition. 2017 signal processing: algorithms, architectures, arrangements, and applications (SPA). New Jersey, USA: IEEE, 263-268, https://doi.org/10.23919/ SPA.2017.8166876.

6. Wang, Z., Li, C., Shao, H. \& Sun, J. (2018). Eye recognition with mixed convolutional and residual network (MiCoRe-Net). IEEE Access, 6, 17905-17912. https://doi.org/10.1109/ACCESS.2018.2812208.

7. Klein, A., Falkner, S., Bartels, S., Hennig, P. \& Hutter, F. (2016). Fast bayesian optimization of machine learning hyperparameters on large datasets. arXiv preprint arXiv:1605.07079.

8. Bazrafkan, S., Thavalengal, S. \& Corcoran, P. (2018). An end to end Deep Neural Network for iris segmentation in unconstrained scenarios. Neural Networks, 106, 79-95, https://doi.org/10.1016/j.neunet.2018.06.011. 
9. Arsalan, M., Naqvi, R. A., Kim, D. S., Nguyen, P.H., Owais, M. \& Park, K. R. (2018). IrisDenseNet: Robust iris segmentation using densely connected fully convolutional networks in the images by visible light and near-infrared light camera sensors. Sensors, 18(5), 1501, https://doi. org/10.3390/s18051501.

10. LeCun, Y., Haffner, P., Bottou, L. \& Bengio, Y. (1999). Object recognition with gradient-based learning. In Shape, contour and grouping in computer vision. Lecture notes in computer science, vol. 1681. Berlin: Springer, 319-345, https://doi.org/10.1007/3-540-46805-6_19.

11. Khan, S., Rahmani, H., Shah, S. A. A. \& Bennamoun, M. (2018). A guide to convolutional neural networks for computer vision. Synthesis lectures on computer vision, vol. 8, no. 1. San Rafael, CA, USA: Morgan \& Claypool Publishers, https://doi.org/10.2200/S00822ED1V01 Y201712COV015.

12. Ciaburro, G. \& Venkateswaran, B. (2017). Neural Networks with $R$ Smart models using CNN, RNN, deep learning, and artificial intelligence principles. Birmingham, UK: Packt Publishing.

13. Russakovsky, O., Deng, J., Su, H., Krause, J., Satheesh, S., Ma, S., et al. (2014). ImageNet large scale visual recognition challenge. Int. J. Comput. Vis., 115(3), 211-252. https://doi.org/10.1007/s11263-015-0816-y.

14. Nguyen, K., Fookes, C., Ross, A. \& Sridharan, S. (2017). Iris recognition with off-the-shelf CNN features: A deep learning perspective. IEEE Access, 6, 18848-18855, https://doi.org/10.1109/ACCESS.2017.2784352.

15. Kim, P. (2017). MATLAB deep learning with machine learning, neural networks and artificial intelligence. New York, USA: Apress.

16. Pan, S. J. \& Yang, Q. (2009). A survey on transfer learning. IEEE Trans. Knowl. Data Eng., 22(10), 1345-1359, https://doi.org/10.1109/ TKDE.2009.191.

17. Zhang, Y., Sohn, K., Villegas, R., Pan, G. \& Lee, H. (2015). Improving object detection with deep convolutional networks via bayesian optimization and structured prediction. 2015 IEEE conference on computer vision and pattern recognition. New Jersey, USA: IEEE, 249258, https://doi.org/10.1109/CVPR.2015.7298621.

18. Goh, G. B., Hodas, N., Siegel, C. \& Vishnu, A. (2018). Smiles2vec: Predicting chemical properties from text representations. 6th International Conference on Learning Representations. Vancouver, BC, Canada, 30 April-3 May, 2018.

19. Institute of Automation, Chinese Academy of Sciences (CASIA). Database. https://biometrics.idealtest.org/. 